\title{
Phenotype of columnar-lined esophagus in rats with esophagogastroduodenal anastomosis: similarity to human Barrett's esophagus
}

\author{
Yinghao $\mathrm{Su}^{1, *}$, Xiaoxin $\mathrm{Chen}^{2, *}$, Michael Klein ${ }^{1}$, Ming Fang ${ }^{1}$, Su Wang ${ }^{2}$, Chung S Yang ${ }^{2}$ \\ and Raj K Goyal ${ }^{1}$ \\ ${ }^{1}$ Center for Swallowing and Motility Disorders, VA Boston Healthcare System and Harvard Medical School, \\ Boston, MA, USA and ${ }^{2}$ Susan Lehman Cullman Laboratory for Cancer Research, Department of Chemical \\ Biology, Ernest Mario School of Pharmacy, Rutgers, The State University of New Jersey, Piscataway, NJ, USA
}

\begin{abstract}
In rats, esophagogastroduodenal anastomosis (EGDA) without concomitant chemical carcinogen treatment can lead to columnar-lined esophagus (CLE) including metaplasia, dysplasia, and esophageal adenocarcinoma (EAC). This study describes the morphology and phenotypic features of CLE and EAC in the rat model and compares them with the corresponding lesions in human Barrett's esophagus (BE). Swiss roll preparations of esophagi of EGDA rats and biopsies from human BE containing specialized intestinal metaplasia (SIM) and EAC were examined. The esophagi of EGDA rats showed esophagitis, CLE, islands of multilayered epithelium (MLE), dysplasia and EAC. The CLE had features of specialized intestinal metaplasia. MLE frequently occurred at the neo-squamocolumnar junction and occasionally in the mid-esophagus in isolated foci. Scattered mucinous cells in esophageal squamous epithelium were also found. The CLE and MLE in EGDA rats resembled the lesions described in human BE in morphology, mucin features and expression of differentiation markers (CK7, CK20, Das-1, villin, and pS2/TFF1). Invasive EAC in EGDA rat is of well-differentiated mucinous type, which is in contrast to the variably differentiated glandular type of adenocarcinoma in human BE. p53, c-myc, and cyclooxygenase 2 are expressed in both the rat and human SIM and EAC. These studies indicate that, not withstanding small differences, SIM and EAC induced in EGDA rats are similar to the corresponding lesions in human BE. EGDA rats may serve as a useful model to study the pathogenesis, molecular biology, and chemopreventive interventions of human BE and EAC.

Laboratory Investigation (2004) 84, 753-765, advance online publication, 19 April 2004; doi:10.1038/labinvest.3700079
\end{abstract}

Keywords: intestinal metaplasia; esophageal adenocarcinoma; phenotype of columnar-lined esophagus

Esophageal adenocarcinoma (EAC) is one of the fastest rising cancers in the Western countries. ${ }^{1}$ The reasons for this alarming increase are not fully understood. Barrett's esophagus (BE) is the main lesion underlying the development of EAC. BE is defined as the replacement of normal squamous epithelium by columnar epithelium, which includes specialized intestinal metaplasia (SIM) as its major constituent. ${ }^{2}$ SIM is characterized by features of type

Correspondence: RK Goyal, MD, VA Medical Center, 1400 VFW Parkway, West Roxbury, MA 02132, USA.

E-mail: raj_goyal@hms.harvard.edu

This work is supported by NIH Grants DK031092 (RK Goyal), DK63650 (X Chen) and CA75683 (CS Yang).

${ }^{*}$ Contributed equally to this publication.

Received 06 November 2003; revised 21 January 2004; accepted 25 January 2004; published online 19 April 2004
III intestinal metaplasia and is the lesion that is associated with the development of EAC. The estimated risk of EAC in BE patients with SIM is around $0.5 \%$ per year which is 30 - to 125 -fold higher than the general population. ${ }^{3} \mathrm{BE}$ is thought to be a complication of gastroesophageal reflux disease, and the development of BE may explain the high association of gastroesophageal reflux disease and EAC. ${ }^{1}$ Despite the clinical importance of BE and EAC, the etiology, cellular origin and pathogenesis of BE and SIM are not well understood. ${ }^{4}$ The roles of gastric acid, bile and other gastrointestinal contents in the pathogenesis of $\mathrm{BE}$ and EAC also remain unclear. Availability of an animal model may help address some of these issues.

Squamous cell carcinoma, adenocarcinoma, and mixed adenosquamous carcinoma are three types of cancer that can be induced in experimental animals. 
Carcinogens such as 2,6-dimethylnitrosomorphine or methyl-n-amylnitrosamine induce esophageal squamous cell carcinoma in experimental animals. On the other hand, gastroduodenal contents also appear to be carcinogenic. Surgical procedures that promote gastroduodenal reflux lead to columnar metaplasia and esophageal adenocarcinomas. ${ }^{5-7}$ When chemical carcinogens are combined with surgically induced gastroesophageal reflux of gastrointestinal contents, mixed adenosquamous carcinomas are generally produced..$^{8,9}$

Goldstein et al ${ }^{10}$ reported that rats with esophagoduodenal anastomosis develop EAC without adenosquamous or squamous cell carcinoma, and the frequency of carcinoma development increases by correction of postoperative anemia with parenteral iron therapy. Similarly, rats surgically treated with esophagogastroduodenal anastomosis (EGDA) to induce esophageal reflux of gastric acid and bile developed columnar-lined esophagus (CLE) and EAC in over half of the animals by 40 weeks after surgery. ${ }^{11}$ The morphological and phenotypic features of the experimental CLE have not been well described. Furthermore, the resemblance of this model to human BE and EAC remains unclear. ${ }^{12}$

Pathological characterization is crucial to the identification of animal models of human disease. ${ }^{13}$ In this study, we describe the morphological features, mucin histochemistry, expression pattern of differentiation markers (CK7, CK20, Das-1, villin, and $\mathrm{pS} 2 / \mathrm{TFF} 1$ ) and cancer-related gene products (p53, c-myc, and cyclooxygenase 2) in CLE and EAC in the EGDA rat model. We then compare the EGDA rat model with findings in human tissues. Our studies show that, in spite of a few differences, there are remarkable similarities of SIM and EAC between the EGDA rats and the human diseases. The EGDA rats may serve as a useful experimental model to study the pathogenesis and cellular origin of human BE. This model may also help in identifying the intermediate molecular markers and developing treatment strategies for human BE and EAC.

\section{Materials and methods}

\section{Animal Models and Tissue Preparation}

The EGDA rats were prepared as previously described. ${ }^{11}$ Briefly, an anastomosis was made between the gastroesophageal junction and the duodenum on the antimesenteric border with accurate mucosal to mucosal opposition in 8-week-old male SpragueDawley rats. The animals were treated with iron ( $4 \mathrm{mg} / \mathrm{kg} /$ week, i.p.) without concomitant carcinogens. They were kept for 40 weeks after surgery and killed by $\mathrm{CO}_{2}$ asphyxiation. The esophagus was removed, fixed in $10 \%$ buffered formalin, Swissrolled, and processed in paraffin. Tissue samples from 20 rats showing both esophagitis and CLE were used for this study. Of them, 14 had visible adenocarcinoma. In total, 10 nonoperated normal rats were used as controls. The Animal Care and Facilities Committee, Rutgers University (protocol \#94-017), approved the protocol for the animal studies. For comparative studies in humans, coded (with all identifiers removed) archival paraffinembedded biopsies having SIM and EAC were obtained from the Department of Pathology at the Veterans Affairs Boston Healthcare System. The study protocol was approved by the Institutional Review Board of the VA Boston Healthcare System (protocol \#1546). Nine tissues had only BE and five had only EAC. Six tissues had both BE and EAC.

\section{Histopathology}

Tissue sections were stained with hematoxylin and eosin (H\&E) for histopathological analysis. Inflammation was graded as mild, moderate, and severe based on the degree of infiltration by the inflammatory cells, particularly neutrophils, eosinophils, macrophages, and plasma cells. The columnar epithelium was graded as normal, reactive or regenerative epithelium, low-grade dysplasia (LGD), and high-grade dysplasia (HGD). ${ }^{14}$ Severe squamous hyperplasia was diagnosed when surface projections of subepithelial papillae caused irregular surface ridges. Inverted, papilloma-like lesions appeared as projections and infolding of the squamous epithelium, without neoplastic changes, into the submucosa and the muscle layer of the esophagus. If small clusters of dysplastic squamous epithelial cells penetrated through the basement membrane into lamina propria and adjacent tissues, the diagnosis of invasive squamous cell carcinoma was made. ${ }^{15}$ However, no case of invasive squamous cell carcinoma was found.

SIM was diagnosed if intestinal columnar epithelium fulfilled features of specialized intestinal metaplasia and contained intestinal-type goblet cells above the anastomotic site marked by the blue prolene suture. Multilayered epithelium (MLE) was diagnosed when the esophageal epithelium showed layers of both squamous and columnar epithelium. ${ }^{16}$ Dysplasia of the columnar mucosa was classified as LGD and HGD, according to the criteria suggested by Haggitt. ${ }^{17}$ In LGD, the crypt architecture trends to be preserved and distortion is minimal; the nuclei may be stratified, but the stratification does not reach the apical surface of the glands; nuclei are enlarged, crowed, and hyperchromatic; mitotic figures may be present in the upper portion of the crypt; goblet and columnar cell mucus is usually diminished or absent, but goblet cells in which the mucous droplet does not communicate with the luminal surface may be observed. The abnormalities extend to the mucosal surface. In HGD, distortion of crypt architecture usually is present and may be marked. It is composed of branching and lateral budding of crypts, a villiform configuration of the mucosal surface or intraglandular bridging of epithelium to 
form a cribriform pattern of 'back-to-back' glands. Nuclear abnormalities are present as in LGD, and stratification reaches the crypt luminal surface. There may be a loss of nuclear polarity, and nuclei often vary markedly in size, shape, and staining characteristics. Goblet and columnar cell mucus is usually absent. The abnormalities extend to the mucosal surface. EAC was diagnosed when dysplastic columnar epithelial cells invaded through the epithelial basement membrane and into deeper tissue.

\section{Histochemistry}

Alcian blue/periodic acid Schiff (AB/PAS) and high iron diamine/Alcian blue (HID/AB) were used for mucin staining. Intestinal metaplasia was classified as complete (type I) or incomplete (type II or type III). All SIM had goblet cells that contained sialomucins and/or sulfomucins. However, in type I intestinal metaplasia, columnar cells had absorptive features but contained no acid mucin; in type II, columnar cells contained sialomucins (stained blue); and in type III intestinal metaplasia, the columnar cells contained sulfomucin (stained purple). ${ }^{18}$ Silver staining (Reticulum Gomori's Silver Kit, Newcomer Supply, Middleton, WI, USA) was used to display the basement membrane of the esophageal epithelium.

\section{Immunohistochemistry}

The paraffin-embedded sections were deparaffinized, rehydrated, and pretreated by $0.1 \%$. trypsin at $37^{\circ} \mathrm{C}$ for $30 \mathrm{~min}$ or by heating slides for $5-10 \mathrm{~min}$ in $10 \mathrm{mM}$ citrate buffer. The staining was performed with the $\mathrm{ARK}^{\mathrm{TM}}$ Peroxidase kit (Dako, Capenteria, CA, USA) according to the manufacturer's instructions. The sources and working concentrations of primary antibodies are as follows: CK7 $(4.1 \mu \mathrm{g} / \mathrm{ml}$, Clone OV-TL 12/30, DAKO), CK20 $(1.2 \mu \mathrm{g} / \mathrm{ml}$, Clone Ks 20.8, DAKO), Das-1 (1:10, a gift kindly provided by $\mathrm{Dr}$ Kiron $\mathrm{M}$ Das, Department of Medicine, Molecular Genetics and Microbiology, University of Medicine and Dentistry of New Jersey), villin $(1.3 \mu \mathrm{g} / \mathrm{ml}$, Clone CWWB1, NeoMarkers, Fremont, CA, USA), pS2/TFF1 $(5 \mu \mathrm{g} / \mathrm{ml}$, Innogenex, San Ramon, CA, USA), p53 $(0.4 \mu \mathrm{g} / \mathrm{ml}$, Clone PAB 240, Chemicon, Temecula, CA, USA), c-myc $(8 \mu \mathrm{g} / \mathrm{ml}$, Clone C-8, NeoMarkers), and COX-2 $(2.5 \mu \mathrm{g} / \mathrm{ml}$, Transduction Lab, Los Angeles, CA, USA). For negative controls, normal serum or phosphatebuffered saline (PBS) replaced primary antibodies. Both positive and negative control slides were processed in parallel.

The staining intensity was subjectively graded into four degrees, from,,-+++ , to +++ according to the following criteria. -, no positive staining; + , less than $2 / 3$ cells with slight staining or less than $1 / 3$ cells with slight to moderate staining; ++ , more than $2 / 3$ cells with slight staining or $1 / 3$ to $2 / 3$ cells with slight to moderate staining; +++ , more than $2 / 3$ cells with moderate or strong staining. Statistical analysis was conducted with Fisher's exact test for frequency data and Ridit analysis for ordinal data. ${ }^{19}$

\section{Results}

\section{Normal Esophageal Mucosa}

Normal esophageal epithelium in the rat is stratified squamous, consisting of a single layer of basal cells with round nuclei, four to six layers of prickle cells, and a thin stratum corneum on the surface. Distally the squamous epithelium is continuous with the squamous epithelium of the forestomach. The rat esophagus has no submucosal glands. This is in contrast to the normal human esophageal epithelium that has stratified squamous epithelium without keratinization and also has submucosal glands. Moreover, in humans, distally the squamous epithelium becomes continuous with the columnar gastric epithelium forming the squamocolumnar $\mathrm{Z}$ line. ${ }^{1}$

\section{Columnar Metaplasia in the Esophagus}

Columnar mucosa is not present in the normal esophagus. Replacement of the normal squamous epithelium with metaplastic columnar epithelium frequently occurred in the EGDA rats. The columnar metaplasia appeared either as a single type CLE or as MLE (columnar and squamous) (Figure 1). These patterns are similar to those found in the human BE.

\section{CLE and Specialized Intestinal Metaplasia}

On H\&E staining, the CLE in EGDA rats consist of a single layer of surface columnar epithelium and tubular mucosal glands. Columnar cells have features of both mucous secretory and absorptive cells with interspersed intestinal-type goblet cells. CLE is present in the distal esophagus near the anastomotic site and is bound by the squamous epithelium of the esophagus and fore-stomach, and the columnar epithelium of the anastomosed duodenum.

In order to define whether the columnar mucosa in the lower esophagus represented the anastomosed duodenal mucosa or metaplastic columnar epithelium of esophageal origin, we examined the neo-squamocolumnar junction and the anastomotic junctions for the continuity of the surface epithelia and the basement membrane. At the anastomotic site, the duodenal and the esophageal or the gastric squamous epithelia as well as their basement membranes were discontinuous. On the other hand, the mucosal junction of the esophageal squamous epithelium and the CLE were continuous without interruption of basement membrane (Figure 2). Moreover, the morphological features of the duodenal mucosa were quite distinct from those of the 


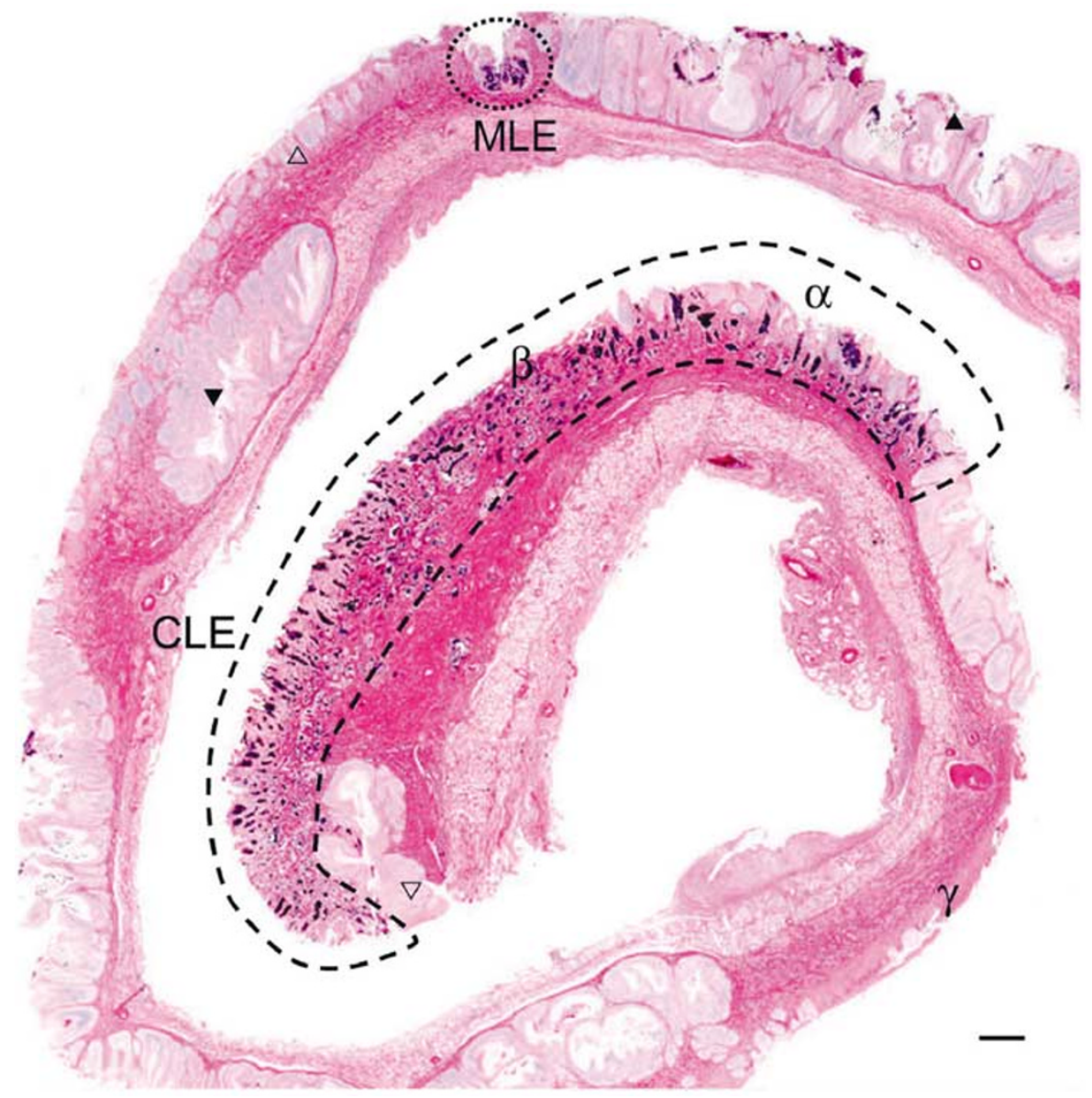

Figure 1 CLE and MLE in an EGDA rat. The figure is montage of a swiss-roll preparation of an EGDA rat esophagus that has been stained with AB/PAS. CLE is demarcated by dashed lines and appears as an area of extensive intestinal metaplasia at the distal end of the esophagus. Note that CLE is continuous with squamous epithelium of the forestomach $(\nabla)$. MLE is demarcated by dotted area in the midesophagus and appears as island of metaplastic epithelium. MLE containing a mixture of squamous and columnar epithelium can also be identified at the neo-squamocolumnar junction $(\alpha)$. Changes in the squamous epithelium including hyperplasia $(\Delta)$, papillary projections $(\boldsymbol{\Delta})$, inverted papilloma-like lesions $(\boldsymbol{\nabla})$, erosions $(\beta)$, and sloughing $(\gamma)$ are frequently observed in the EGDA rats. Scale bar: $200 \mu \mathrm{m}$.

CLE. These observations are consistent with the view that the columnar mucosa lining the esophagus was metaplastic epithelium of esophageal origin. In some cases, CLE was covered by squamous epithelium as has been described in humans, and may be related to partial healing of BE. All of the 20 cases of CLE had intestinal metaplasia, including 17 cases of type III and three cases of type I. There were 14 cases of dysplasia, including 12 LGD and two HGD (Table 1).

On mucin staining (PAS/AB and HID/AB), CLE in the EGDA rats shows that the goblet cells contained both sialomucin and sulfomucin, whereas the columnar cells stain for neutral mucin, sialomucin or sulfomucin. The staining intensity of sulfomucin is similar to that of sialomucin in 15 of 20 cases. The mucin stains clearly distinguished CLE from the rat duodenal mucosa, which showed sulfomucin in the goblet cells but not the columnar cells. ${ }^{20}$ However, the mucin staining features of CLE in the rat (Figure 3a) and human BE (Figure 3b) were similar, resembling the type III intestinal metaplasia in most cases. In order to characterize further the phenotype of SIM in the EGDA rats, we examined for the presence of several other markers and compared the results with similar studies in human BE (Table 2). The CK7/CK20 pattern of superficial CK20 staining in surface glands and diffuse CK7 staining in surface and deep glands has been attributed to be specific to BE. ${ }^{21}$ This pattern was observed in three of 20 rat CLE (Figure 3c, e) and 11 of 15 human BE (Figure 3d, f) (Fisher's: $P<0.05$ ). Other staining patterns, such as diffuse CK7/diffuse CK20 (one rat and one human), superficial CK7/ superficial CK20 (one rat and one human), and diffuse CK7/negative CK20 (one rat and two humans) were also observed. Four cases of rat SIM had diffuse expression of CK20 and some scattered CK7positive cells. The remaining 10 cases of rat SIM did not express either CK7 or CK20.

Das-1, a polyclonal antibody, which recognizes normal human colon epithelium and $\mathrm{BE}$ in the gastrointestinal tract, ${ }^{22}$ positively stained nine of 20 rat CLE and 14 of 15 human BE (Fisher's: $P<0.05$ ). 

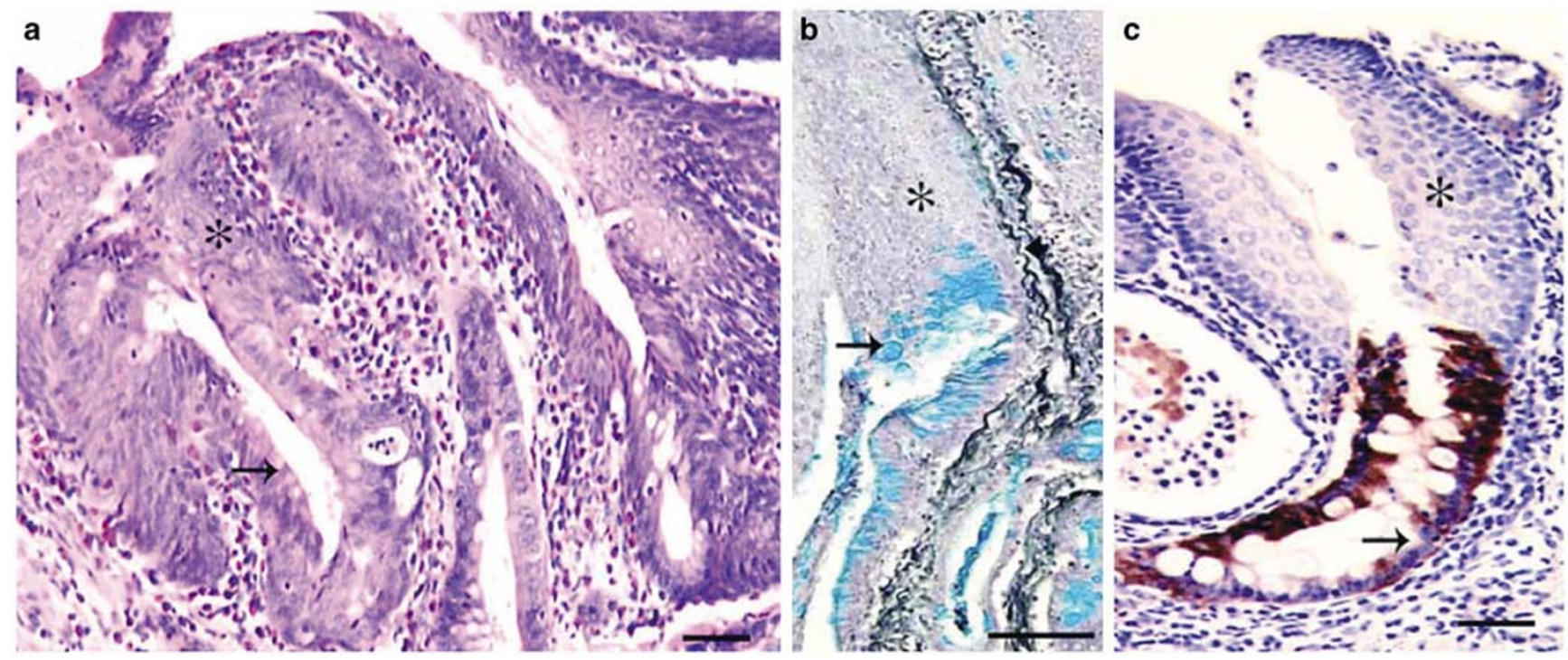

Figure 2 Showing metaplastic nature of the columnar mucosa in EGDA rats. (a) H\&E stain of the neo-squamocolumnar junction at the distal end of the esophagus in an EGDA rat. Note that the metaplastic columnar epithelium $(\rightarrow)$ is interposed between areas of squamous epithelium on either side. (b) Gomori silver-Alcian blue-hematoxylin triple staining showing that the basement membrane (short black arrow) between the squamous and columnar epithelia is not interrupted, thus arguing against the mucosal junction being a surgical approximation of duodenal and esophageal mucosa. (c) Represents villin immunostaining of the neo-squamocolumnar junction. The columnar epithelium shows villin immunoreactivity (dark brown), and squamous epithelium is negative for villin immunostaining. Note the direct continuity of the metaplastic columnar epithelium with the squamous epithelium. Scale bars: $100 \mu \mathrm{m}$.

Table 1 Histopathology of esophageal mucosa in the rat model and human BE

\begin{tabular}{lcc}
\hline & $\begin{array}{c}\text { Rat model } \\
\text { No. of cases } \\
(\%)\end{array}$ & $\begin{array}{c}\text { Human BE } \\
\text { No. of cases } \\
(\%)\end{array}$ \\
\hline $\begin{array}{l}\text { Total no. examined } \\
\text { Squamous epithelium }\end{array}$ & & \\
$\begin{array}{l}\text { Regenerative } \\
\text { Low-grade dysplasia }\end{array}$ & 20 & 15 \\
High-grade dysplasia & $4(20)$ & $6(50)^{\mathrm{c}}$ \\
Surface ridges & $12(60)$ & $6(50)$ \\
Inverted papilloma-like lesion & $4(20)$ & 0 \\
Inflammatory responseb & $2(10)$ & 0 \\
$\quad$ None & $7(35)$ & 0 \\
Mild & & \\
Moderate & 0 & 0 \\
Severe & $4(20)$ & $6(40)$ \\
Intestinal metaplasia & $12(60)$ & $9(60)$ \\
Type I & $4(20)$ & 0 \\
Type II & $3(15)$ & $3(20)$ \\
$\quad$ Type III incomplete & 0 & 0 \\
Dysplasia & $17(85)$ & $12(80)$ \\
None & & \\
LGD & $6(30)$ & $3(20)$ \\
HGD & $12(60)$ & $6(40)$ \\
& $2(10)$ & $6(40)$
\end{tabular}

${ }^{\mathrm{a}}$ Ridit: $\quad \mu=2.19 \quad(P<0.01)$; ${ }^{\mathrm{b}}$ Ridit: $\quad \mu=1.8 \quad(P>0.05)$; $\quad{ }^{\mathrm{c}}$ Squamous epithelium was absent in three human tissues.

The cytoplasmic immunostaining of Das-1 was weaker in the rat CLE (Figure 3g) than in human BE (Figure 3h). Villin, a cell differentiation marker for intestinal brush-border formation, ${ }^{23,24}$ was observed frequently in rat SIM than in human SIM without significant difference (Fischer's: $P>0.05$ ).
The cytoplasmic immunostaining of villin was usually stronger in the rat (Figure 3i) than in the human (Figure 3j) tissues. The cytoplasmic staining of pS2/TFF1, a marker of ulcer-associated cell lineage, ${ }^{25}$ was found in 17 of 20 rat CLE (Figure $3 \mathrm{k}$ ) and 13 of 15 human BE (Figure 3l) with similar frequency (Fisher's: $P>0.05$ ) and immunoreactivity.

\section{Multilayered epithelium}

In three cases of EGDA rats, scattered mucinous cells appeared admixed with hyperplastic squamous epithelium (Figure 4a). In three other cases, MLE was identified as islands of mucinous cells interspersed in squamous epithelium (Figure 4b). The columnar and goblet cells may appear on the top, in the middle, or at the bottom of the squamous cell component of MLE. One case had mucinous cells located at the superficial squamous epithelium far removed from the anastomosis site (Figure 1). Two cases had mucinous cells located in deep nests of hyperplastic squamous epithelium in the neosquamocolumnar junction. Multiple foci of MLE were found in one case and presented as three separate foci distributed in distinct nests of hyperplastic squamous epithelium. In our biopsies of human tissue, MLE was not found. This is not surprising because this material represented directed biopsies of the CLE. In humans, MLE is found only in random biopsies taken at the squamocolumnar junction. The morphologic feature of MLE in the rat model closely resemble those described in the human patients. ${ }^{16,26}$ 

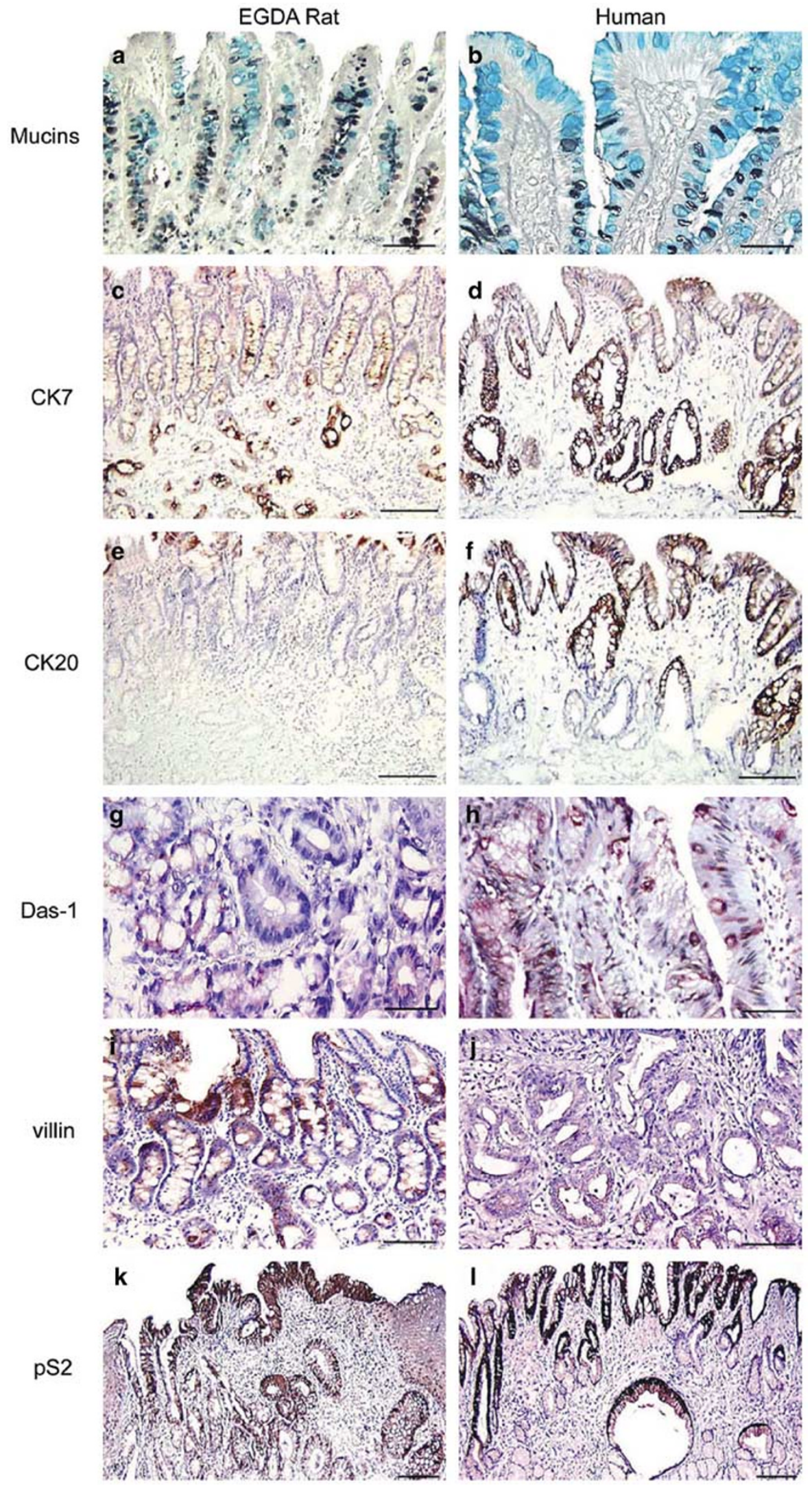
Table 2 Differentiation and cancer-related markers in interstitial metaplasia and adenocarcinoma in the rat model and human BE

SIM No. of cases (\%) EAC No. of cases (\%)

\begin{tabular}{|c|c|c|c|c|}
\hline & $\begin{array}{c}\text { Rat } \\
\text { model }\end{array}$ & $\begin{array}{l}\text { Human } \\
\qquad E\end{array}$ & $\begin{array}{c}\text { Rat } \\
\text { model }\end{array}$ & $\begin{array}{c}\text { Human } \\
\text { BE }\end{array}$ \\
\hline Total no. of tissues & 20 & 15 & 14 & 11 \\
\hline \multicolumn{5}{|c|}{ Differentiation markers } \\
\hline CK7 & $10(50)$ & $15(100)^{\mathrm{a}}$ & $4(29)$ & $10(91)^{\mathrm{a}}$ \\
\hline CK20 & $9(45)$ & $13(87)^{\mathrm{a}}$ & $4(29)$ & $9(82)^{a}$ \\
\hline CK BE pattern & $3(15)$ & $11(73)^{\mathrm{a}}$ & NA & NA \\
\hline DAS-1 & $9(45)$ & $14(93)^{\mathrm{a}}$ & $1(7)$ & $8(73)^{\mathrm{a}}$ \\
\hline Villin & $13(65)$ & $5(33)$ & $9(64)$ & $3(27)$ \\
\hline PS2/TFF1 & $17(85)$ & $13(87)$ & $13(93)$ & $6(55)$ \\
\hline \multicolumn{5}{|c|}{ Cancer-related markers } \\
\hline P53 & $15(75)$ & $10(67)$ & $4(29)$ & $3(27)$ \\
\hline c-myc & $5(25)$ & $2(13)$ & 0 & $3(27)$ \\
\hline COX-2 & $16(80)$ & $3(20) a$ & $7(50)$ & $2(18)$ \\
\hline
\end{tabular}

${ }^{\mathrm{a}}$ Fisher's exact test, significantly different from the rat model $(P<0.05)$.

NA, not applicable.

We also examined the phenotypic characters of the columnar cells of MLE in the rat model. Mucinous cells in the rat model contained both sulfomucin and sialomucin, but little to no neutral mucin (Figure 4c, d). The foci of MLE were readily detected with AB/PAS staining. The mucinous cells of rat MLE in the deep nests and surface of hyperplastic squamous epithelium showed strong immunoreactivity of CK7, moderate to strong staining of villin, weak staining of Das- 1 and pS2/TFF1, and negative staining of CK20 (Figure 4e-h). These biochemical markers in the rat are similar to those described in humans. ${ }^{16}$

\section{Esophageal Adenocarcinoma}

All of the 14 cases of EAC in EGDA rats were localized to the esophagus just above the esophagogastroduodenal anastomosis. Two cancers were early cancers limited to the submucosa. In total, 12 cases were advanced EAC showing invasion of cancer cells into muscle, adventitia, and adjacent tissues (Figure 5a-c). These cancers clearly arose from the SIM in the esophagus rather than from the duodenal mucosa (Figure 5d). EACs in the rat model were highly differentiated mucinous carcinomas. In contrast, 11 cases of human EAC showed various degrees of differentiation (well-46\%, moderately$27 \%$, and poorly-27\%) with glandular structures in 10 cases and mucinous morphology in only one case.

Similar to adjacent CLE, rat EAC had large quantities of sulfomucin and sialomucin, and lesser amounts of neutral mucin (Figure 5e). CK7 (Figure 5f), CK20, and Das-1 were less frequently expressed in rat EAC than in human EAC (Table 2). Rat EAC also expressed villin (Figure $5 \mathrm{~g}$ ) and $\mathrm{pS} 2 / \mathrm{TFF} 1$.

Accumulation of the tumor suppressor p53 protein was found in $29 \%(4 / 14)$ of EAC and $75 \%(15 /$ 20) of CLE in EGDA rats (Fisher's: $P<0.05$ ). The p53 protein was localized in the nuclei and cytoplasm, mainly in the deep glands of the neo-squamocolumnar junction (Figure 6a). Cytoplasmic staining of oncogene $c$-myc was found in the CLE $(5 / 20,25 \%)$, but not EAC, of EGDA rats (Figure 6b). The epithelial COX-2 immunoreactivity was seen more frequently in rat CLE than in human BE (Figure 6c) (Table 2). Hyperplastic squamous epithelium, fibroblasts, and infiltrated inflammatory cells also showed weak to strong COX-2 immunoreactivity in both EGDA rats and human patients.

\section{Changes in Esophageal Squamous Epithelium in the Rat Model}

Esophageal squamous epithelium of EGDA rats exhibited various degrees of mucosal erosion, ulceration, and infiltration of acute inflammatory cells, both eosinophils and neutrophils. Basal cell hyperplasia was so severe that it often occupied the entire thickness of the mucosa. Projections of fibrovascular papillae with a thin core formed mucosal ridges. A prominent feature was inverted squamous papilloma-like lesions in which rete pegs extended down into the submucosa to the muscle (Figure 1). Inverted papilloma-like lesions have not previously been described in the esophagi of humans or experimental animals, but have been well described in the nasal mucosa and the urinary bladder. ${ }^{27}$ Single or multiple inverted papillomalike lesions were found in seven out of 20 EGDA rats. Unlike squamous cell carcinoma, inverted

Figure 3 Comparison of mucin staining and immunohistochemical features of rat CLE and human BE. (a, b) HID/AB staining show that goblet cells of CLE in EGDA rats (a) contain both sialomucin (blue) and sulfomucin (dark brown or black), whereas columnar cells stain for sulfomucin. This feature is similar to that seen in human BE (b). (c-f) show CK7/CK20 immunostaining in rat CLE and human disease. In the rat CLE, CK7 immunoreactivity is present in both the superficial crypts and the deep gland epithelium (c); CK20 immunoreactivity is present only in the superficial epithelium and crypts (e). This CK7/CK20 immunostaining pattern is similar to the BE-specific CK7/ CK20 pattern in human BE (d, f). (g, h) show Das-1 immunostaining which was seen in both the animal model and in human tissues. However, cytoplasmic staining of Das-1 is weaker in EGDA rats (g) than in human BE (h). As shown in (i, $\mathbf{j})$ cytoplasmic staining of villin is also observed in the metaplastic epithelium in EGDA rats as well as in human BE tissues. However, villin staining is stronger in EGDA rats (i) than in human BE (j). A similar expression pattern of pS2/TFF1 was found in rat CLE (k) and human BE (l). In both cases, superficial and upper glandular epithelium shows strong immunostaining of pS2/TFF1. Scale bars: (a, b, g and h) $100 \mu \mathrm{m} ;(\mathbf{c}-\mathbf{f}, \mathbf{i}, \mathbf{j})$ $150 \mu \mathrm{m}$; (k, l) $200 \mu \mathrm{m}$. 

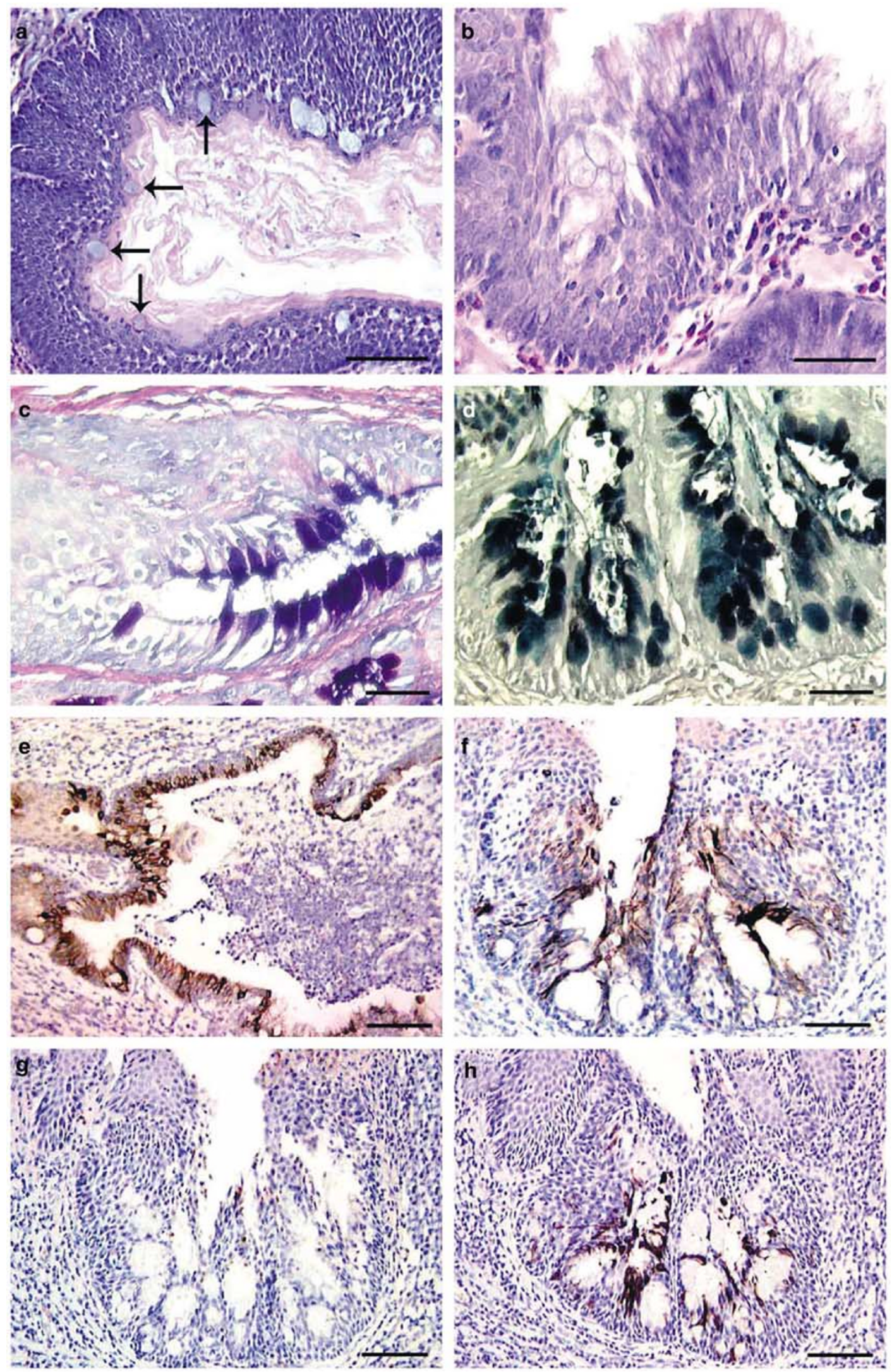
papillary extensions neither have irregular edges nor dysplastic cells spreading into the adjacent structures. No invasive squamous cell carcinoma was found. The squamous mucosa from human patients with BE showed mild-to-moderate basal cell hyperplasia but intramural papillary extensions were not observed.

\section{Discussion}

This study shows that rats with esophagogastroduodenal anastomosis (EGDA) develop: (1) SIM that has phenotypic features similar to those found in human Barrett's esophagus; (2) MLE that has recently been described in human disease; and (3) EAC. This study suggests that EGDA rats may serve as a suitable model for investigations of pathophysiology, molecular biology of progression to cancer, and prevention and treatment trials of human Barrett's esophagus.

The esophageal mucosal changes in EGDA rats are likely to be secondary to the reflux of gastroduodenal contents into the esophagus. Although, we did not perform quantitative measurement of gastric acid duodenal contents or bile in the esophagus of these animals, presence of free bile reflux in the EGDA rats was evident by bile staining of the esophageal mucosa. Bile staining of esophageal mucosa was not seen in normal control. In a related animal model, increased bile acids have been described in the esophageal aspirate of rats after esophagojejunostomy. ${ }^{6,28}$ Other investigators have described that duodenal or gastroduodenal contents, particularly deoxycholic acid may lead to esophageal adenocarcinoma ${ }^{7}$ by activating NF-Kappa-B and inducing IL-8 expression. ${ }^{29,30}$ Role of gastric acid in pathogenesis of esophageal adenocarcinoma is unclear. ${ }^{31,32}$

The morphology and mucin histochemistry of the columnar metaplasia in the rat model are identical to those that characterize the specialized intestinal metaplasia in human Barrett's esophagus, including mucous-containing columnar cells that have both secretory and absorptive features and intestinal type of goblet cells that contain sulfomucins. These features are also similar to what has been described as type III intestinal metaplasia. The SIM in the rat model also expresses phenotypic markers that are seen in SIM in human Barrett's esophagus, including the expression of differentiation markers
(Figure 2). The existence of BE-specific CK pattern, colon-specific antigen (Das-1), villin and a marker of ulcer associated cell lineage (pS2/TFF1) ${ }^{33}$ in the metaplastic epithelium in the rat is similar to that seen in humans suggesting that SIM in rats and humans may have similar cellular origin. However, differentiation markers such as CK7/CK20 and Das-1 were less often expressed in the rat than in the human tissues. The reason for this difference is not clear. This may be due to lower affinity of rat tissues than human tissues to these antibodies that were raised against human antigens. Further studies are needed to resolve this important point.

MLE, consisting of multiple layers of squamous and columnar cells, was described by Shields and colleagues, who reported it in $41 \%$ of mucosal biopsies from the squamocolumnar junction in patients with BE. All the samples contained goblet cells. ${ }^{26}$ We found 4/20 cases of MLE at the neosquamocolumnar junction in the EGDA rats. One of the most interesting findings of this study was the discovery of MLE in the mid-esophagus of two EGDA rats after careful search in Swiss-roll preparations of the esophagus. The low frequency of MLE in the mid-esophagus may be due to the relatively mild reflux injury to the mucosa compared to the distal esophagus. Furthermore, MLE in the mid-esophagus may go undetected unless serial tissue sections are stained with $\mathrm{AB} / \mathrm{PAS}$ and examined carefully. It is likely that MLE has not yet been described in the mid-esophagus of human for the same reasons. We speculate that careful examination of tissues from BE patients suffering from severe gastroesophageal reflux may reveal isolated islands of MLE and scattered mucinous cells high up in the esophagus, away from the gastroesophageal junction.

MLE in humans expresses cytokeratin markers of both squamous (CK4 and CK13) and columnar epithelium (CK7 and CK20), suggesting that it may be a transitional stage between squamous epithelium and BE. ${ }^{16,34}$ It exhibits a certain frequency of mucin expression (neutral mucin, sulfomucin and sialomucin in 88,71 and $100 \%$, respectively) and expresses differentiation markers such as villin and pS2/TFF1. In our study, MLE in the rat model was found to share features with human MLE, except lesser neutral mucins and no CK20 were expressed in the rat model. Overall, this study suggests that MLE in the EGDA rat, as in humans, may be an intermediate stage between esophageal squamous

Figure 4 Histochemical and immunohistochemical features of MLE in EGDA rats. (a, b) H\&E staining of an area of MLE showing scattered mucinous cells on the top of hyperplastic squamous cells (a). In typical MLE, mucinous cells including columnar and goblet cells are lined up on top of the squamous component (b). (c) AB/PAS staining with hematoxylin counterstaining shows that the mucinous cells mainly secrete acid mucins (blue or purple) with little to no neutral mucin (red). (d) HID/AB staining shows that both sialomucin and sulfomucin are present in the mucinous cells. (e, f) shows CK7 immunoreactivity of the MLE. Note that the mucinous cells in the deep nests of hyperplastic squamous epithelium (e) and surface esophageal mucosa (f) demonstrate strong CK7 immunoreactivity. (g) The MLE in rats shows no staining for CK20. (h) shows that a strong cytoplasmic immunostaining of villin is found in the mucinous cells of MLE in rats. Scale bars: (a-d) $50 \mu \mathrm{m}$; (e-h) $100 \mu \mathrm{m}$. 
762
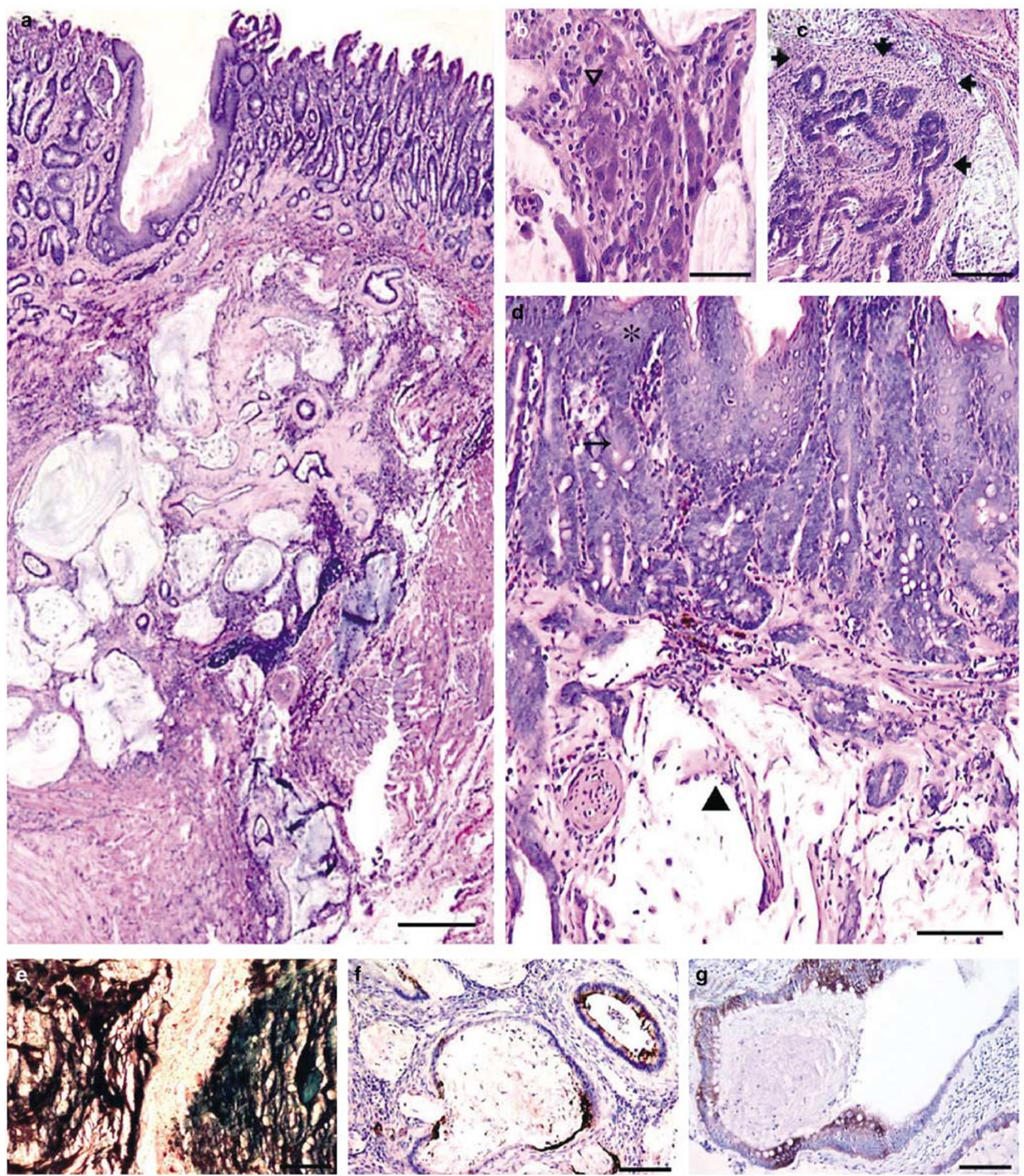

Figure 5 Histological characteristics of EAC in EGDA rats. (a) H\&E staining shows that EAC consists of dilated, cystic glands with abundant mucin secretion and epithelial dysplasia. The mucinous glands can be seen invading through the muscle layers. (b) shows invasion of EAC into adjacent liver tissue. Note that residual hepatocytes $(\nabla)$ are seen in the stroma of EAC. (c) shows invasion of EAC cells in an adjacent nerve. Note that the invasive cancer cells are breaking through the epineurium and endoneurium of the nerve (short black arrow). (d) shows continuity of the EAC $(\boldsymbol{\Delta})$ with the CLE $(\rightarrow)$ in the mucosa. (e) HID/AB staining shows large quantities of sulfomucin (dark brown) and sialomucin (blue) in the extracellular mucinous 'lakes' are in the rat EAC. (f) shows that EAC cells stain for CK7 and (g) shows cytoplasmic staining of villin in EAC. Scale bars: (a) $200 \mu \mathrm{m}$; (b-g) $100 \mu \mathrm{m}$. 

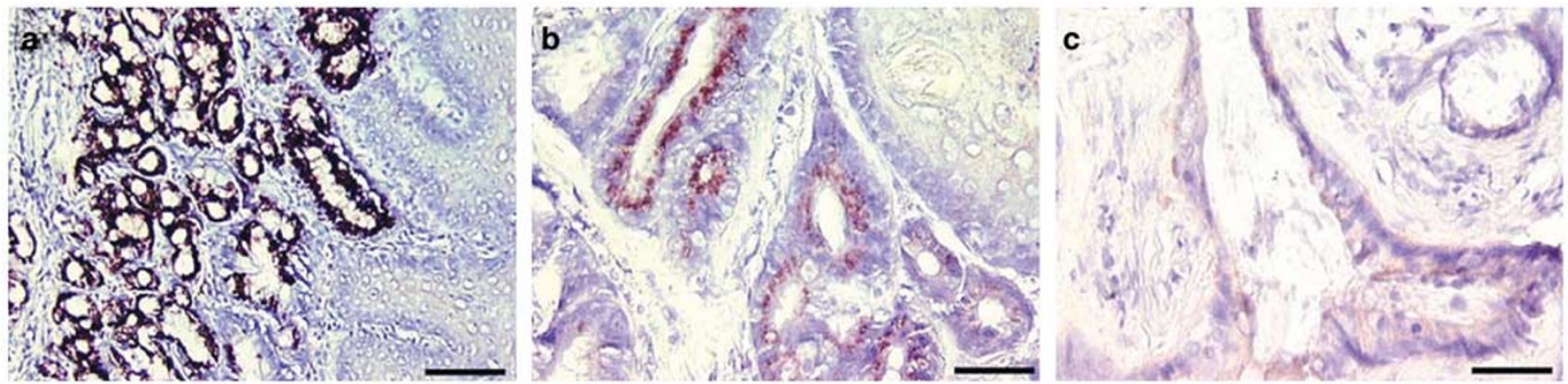

Figure 6 Expression of p53, c-myc and COX-2 in the rat CLE and EAC. (a) shows that CLE in the deep glands exhibit strong nuclear and cytoplasmic staining for p53. (b) shows intermediate cytoplasmic staining of c-myc in areas of dysplasia, and (c) shows a weak cytoplasmic staining of COX-2 in EAC . Scale bars, $50 \mu \mathrm{m}$.

epithelium and SIM. The presence of multiple MLE foci and scattered mucinous cells in the esophagi of EGDA rats also suggest that SIM may arise from different clones of the pluripotential stem cells in the esophagus.

In human patients with BE, SIM and MLE have been proposed to arise from progenitor cells in esophageal submucosal glandular ducts or the transitional cell zone at the gastroesophageal junction. ${ }^{16,35}$ The rat, however, does not have submucosal glands in the esophagus nor does it have the transitional cell zone between esophageal squamous epithelium and gastric columnar epithelium that are seen in humans. (In the rat the fore-stomach is lined by squamous epithelium.) Instead, EGDA rats have a neo-squamocolumnar junction between esophageal/ gastric squamous epithelium and duodenal columnar epithelium. It was previously suggested that CLE in the rat model was derived from the creeping substitution of esophageal mucosa by the duodenal mucosa. ${ }^{11}$ However, the histological features and mucin histochemistry in SIM was quite different from the duodenal mucosa. Moreover, the phenotypic markers of differentiation in the SIM or MLE at the neosquamocolumnar junction and in the mid-esophagus do not support the view that the CLE in the animal model represents creeping substitution of the duodenal mucosa. Instead, our studies suggest that SIM in the animal model may arise from pluripotential stem cells in the esophageal squamous epithelium. ${ }^{7}$ Studies of gene expression profiles using gene chips may further define the cellular origin of the specialized intestinal metaplasia.

The EGDA rats showed severe changes in the squamous epithelium consisting of hyperplasia with ridge-like surface projections and inverted papilloma-like lesions. These lesions did not show features of neoplasia. Such lesions have been described in human nasal mucosa but not in the human esophagus. The reason for this severe squamous epithelial reaction in the EGDA rats but not in human SIM is not clear. However, it may be related to the more acute and severe reflux injury in the animal model than in clinical conditions in humans.
The EGDA rats also showed changes of dysplasia in the metaplastic epithelium and developed adenocarcinomas, as seen in the human disease. Similar to humans, all EACs in the rat were in the distal esophagus. The mucin features, differentiation markers such as CK7, Das-1, villin and pS2/TFF1, and tumor markers such as p53 and c-myc in the cells of EAC were similar to those in the adjacent SIM. These findings strongly suggest that EAC in EGDA rats arises from the SIM in the esophagus and is not an unrelated adenocarcinoma arising from the small bowel, as has been suggested by some investigators. ${ }^{12}$

As described earlier, all the EACs in the rat model were well-differentiated mucinous adenocarcinomas. ${ }^{5,10-12}$ In patients with BE variably differentiated adenocarcinomas are seen. In our study, only one of 11 human EACs was of the mucinous type. The reason for these differences in the histological types of adenocarcinomas in the rat and human is not currently known. However, this difference may be due to differences in the expression of certain differentiation genes. ${ }^{33}$

The animal models of esophagoduodenal and esophagogastroduodenal reflux have been used to understand the pathogenesis of SIM and EAC. It has been reported that oxidative stress augmented by iron overload is an important risk factors for the development of EAC in the EGDA rats. ${ }^{36,37}$ Moreover, abnormalities in arachidonic acid have been reported to be important in the pathogenesis of SIM and EAC. ${ }^{38}$ A recent study has also demonstrated the efficacy of COX-2 inhibitors in the prevention of EAC in rats with esophagoduodejejunostomy. ${ }^{39}$ The present study, showing phenotypic similarities between the lesions in the EGDA rats and in patients with $\mathrm{BE}$, suggests that conclusions drawn from the animal studies may be relevant to clinical BE.

Similar to humans, SIM in the EGDA rats was accompanied by LGD and HGD with a high prevalence of nuclear accumulation of p53 protein $(15 / 20,75 \%)$. Fein et $a l^{40}$ reported that jejunoesophagostomy in p53-knockout mice promoted the development of columnar metaplasia and EAC in 
mice ${ }^{40}$ It suggests that p53 protein accumulation is an important early event in the pathogenesis of rat EAC, as in humans.

In conclusion, SIM and EAC developed in the EGDA rats are remarkably similar to human BE and EAC. The EGDA rat model may serve as a useful experimental system for studying the pathogenesis of $\mathrm{BE}$ and the progression of BE to EAC. It has been shown that concurrent treatment with certain chemical carcinogens promotes development of squamous cell carcinoma or adenosquamous carcinoma in the animal model of gastroduodenal reflux. ${ }^{8,9}$ Further studies in this model may help in understanding the molecular pathways that selectively lead to one or the other type of esophageal carcinoma. The animal model may also be helpful in tracing changes in signaling pathways involved in the pathogenesis of esophageal adenocarcinomas and in identifying intermediate markers that may predict progression of SIM to EAC. In addition, the EGDA rat model can be instrumental for investigating strategies for the prevention and treatment of human Barrett's esophagus and EAC. ${ }^{37}$

\section{Acknowledgements}

We thank Dr Kiron M Das for providing the polyclonal antibody Das-1, Dr Howard Chang and Dr Lingxin Kong for helpful suggestions.

\section{References}

1 Spechler SJ, Goyal RK. The columnar-lined esophagus, intestinal metaplasia, and Norman Barrett. Gastroenterology 1996;110:614-621.

2 Paull A, Trier JS, Dalton MD, et al. The histologic spectrum of Barrett's esophagus. N Engl J Med 1976; 295:476-480.

3 Shaheen NJ, Crosby MA, Bozymski EM, et al. Is there publication bias in the reporting of cancer risk in Barrett's esophagus? Gastroenterology 2000;119:333-338.

4 Falk GW. Barrett's esophagus. Gastroenterology 2002; 122:1569-1591.

5 Fein M, Peters JH, Chandrasoma P, et al. Duodenoesophageal reflux induces esophageal adenocarcinoma without exogenous carcinogen. J Gastrointest Surg 1998;2:260-268.

6 Miwa K, Sahara H, Segawa M, et al. Reflux of duodenal or gastro-duodenal contents induces esophageal carcinoma in rats. Int J Cancer 1996;67:269-274.

7 Pera M, Brito MJ, Poulsom R, et al. Duodenal-content reflux esophagitis induces the development of glandular metaplasia and adenosquamous carcinoma in rats. Carcinogenesis 2000;21:1587-1591.

8 Attwood SE, Smyrk TC, DeMeester TR, et al. Duodenoesophageal reflux and the development of esophageal adenocarcinoma in rats. Surgery 1992;111:503-510.

9 Pera M, Cardesa A, Bombi JA, et al. Influence of esophagojejunostomy on the induction of adenocarcinoma of the distal esophagus in Sprague-Dawley rats by subcutaneous injection of 2,6-dimethylnitrosomorpholine. Cancer Res 1989;49:6803-6808.
10 Goldstein SR, Yang GY, Curtis SK, et al. Development of esophageal metaplasia and adenocarcinoma in a rat surgical model without the use of a carcinogen. Carcinogenesis 1997;18:2265-2270.

11 Chen X, Yang G, Ding WY, et al. An esophagogastroduodenal anastomosis model for esophageal adenocarcinogenesis in rats and enhancement by iron overload. Carcinogenesis 1999;20:1801-1808.

12 Oberg S, Lord RV, Peters JH, et al. Is adenocarcinoma following esophagoduodenostomy without carcinogen in the rat reflux-induced? J Surg Res 2000;91:111-117.

13 Boivin GP, Washington K, Yang K, et al. Pathology of mouse models of intestinal cancer: consensus report and recommendations. Gastroenterology 2003;124: 762-777.

14 Schlemper RJ. International consensus classification of gastrointestinal epithelial neoplasia: usefulness for esophageal squamous epithelium. In: Imamura $\mathrm{M}$ (ed). Superficial Esophageal Neoplasm: Pathology, Diagnosis, and Therapy. Springer-Verlag: Tokyo, Japan, 2002, pp 75-82.

15 Rubio CA. Histocytologic criteria for squamous cell carcinoma in situ of the esophagus. In: Imamura M (ed). Superficial Esophageal Neoplasm: Pathology, Diagnosis, and Therapy. Springer-Verlag: Tokyo, Japan, 2002, pp 113-120.

16 Glickman JN, Chen YY, Wang HH, et al. Phenotypic characteristics of a distinctive multilayered epithelium suggests that it is a precursor in the development of Barrett's esophagus. Am J Surg Pathol 2001;25:569-578.

17 Haggitt RC. Barrett's esophagus, dysplasia, and adenocarcinoma. Hum Pathol 1994;25:982-993.

18 Jass JR, Filipe MI. The mucin profiles of normal gastric mucosa, intestinal metaplasia and its variants and gastric carcinoma. Histochem J 1981;13:931-939.

19 Stokes ME, Davis CS, Koch GG. Categorical Data Analysis using the SAS System. SAS Institute, Inc: Cary, NC, 2000.

20 Sheahan DG, Jervis HR. Comparative histochemistry of gastrointestinal mucosubstances. Am J Anat 1976;146: 103-131.

21 Ormsby AH, Vaezi MF, Richter JE, et al. Cytokeratin immunoreactivity patterns in the diagnosis of shortsegment Barrett's esophagus. Gastroenterology 2000; 119:683-690.

22 Das KM, Prasad I, Garla S, et al. Detection of a shared colon epithelial epitope on Barrett epithelium by a novel monoclonal antibody. Ann Intern Med 1994;120: 753-756.

23 MacLennan AJ, Orringer MB, Beer DG. Identification of intestinal-type Barrett's metaplasia by using the intestine-specific protein villin and esophageal brush cytology. Mol Carcinogen 1999;24:137-143.

24 Glickman JN, Wang H, Das KM, et al. Phenotype of Barrett's esophagus and intestinal metaplasia of the distal esophagus and gastroesophageal junction: an immunohistochemical study of cytokeratins 7 and 20, Das-1 and 45 MI. Am J Surg Pathol 2001;25:87-94.

25 Poulsom R, Wright NA. Trefoil peptides: a newly recognized family of epithelial mucin-associated molecules. Am J Physiol 1993;265:G205-G213.

26 Shields HM, Rosenberg SJ, Zwas FR, et al. Prospective evaluation of multilayered epithelium in Barrett's esophagus. Am J Gastroenterol 2001;96:3268-3273.

27 Witjes JA, van Balken MR, van de Kaa CA. The prognostic value of a primary inverted papilloma of the urinary tract. J Urol 1997;158:1500-1505. 
28 Fein M, Fuchs KH, Stopper H, et al. Duodenogastric reflux and foregut carcinogenesis: analysis of duodenal juice in a rodent model of cancer. Carcinogenesis 2000;21:2079-2084.

29 Jenkins GJ, Harries K, Doak SH, et al. The bile acid deoxycholic acid (DCA) at neutral $\mathrm{pH}$, activates NFkappaB and induces IL-8 expression in oesophageal cells in vitro. Carcinogenesis 2003, Dec 4 [Epub ahead of print].

30 Tselepis C, Morris CD, Wakelin D, et al. Upregulation of the oncogene c-myc in Barrett's adenocarcinoma: induction of c-myc by acidified bile acid in vitro. Gut 2003;52:174-180.

31 Ireland AP, Peters JH, Smyrk TC, et al. Gastric juice protects against the development of esophageal adenocarcinoma in the rat. Ann Surg 1996;224: 358-370.

32 Kawaura Y, Tatsuzawa Y, Wakabayashi $\mathrm{T}$, et al. Immunohistochemical study of p53, c-erbB-2, and PCNA in Barrett's esophagus with dysplasia and adenocarcinoma arising from experimental acid or alkaline reflux model. J Gastroenterol 2001;36: 595-600.

33 Warson C, Van De Bovenkamp JH, Korteland-Van Male AM, et al. Barrett's esophagus is characterized by expression of gastric-type mucins (MUC5AC, MUC6) and TFF peptides (TFF1 and TFF2), but the risk of carcinoma development may be indicated by the intestinal-type mucin, MUC2. Hum Pathol 2002; 33:660-668.

34 Boch JA, Shields HM, Antonioli DA, et al. Distribution of cytokeratin markers in Barrett's specialized columnar epithelium. Gastroenterology 1997;112:760-765.

35 Jankowski JA, Harrison RF, Perry I, et al. Barrett's metaplasia. Lancet 2000;356:2079-2085.

36 Chen X, Ding YW, Yang G, et al. Oxidative damage in an esophageal adenocarcinoma model with rats. Carcinogenesis 2000;21:257-263.

37 Chen X, Yang CS. Esophageal adenocarcinoma: a review and perspectives on the mechanism of carcinogenesis and chemoprevention. Carcinogenesis 2001;22:1119-1129.

38 Chen X, Li N, Wang S, et al. Aberrant arachidonic acid metabolism in esophageal adenocarcinogenesis, and the effects of sulindac, nordihydroguaiaretic acid, and alpha-difluoromethylornithine on tumorigenesis in a rat surgical model. Carcinogenesis 2002;23: 2095-2102.

39 Buttar NS, Wang KK, Leontovich O, et al. Chemoprevention of esophageal adenocarcinoma by COX-2 inhibitors in an animal model of Barrett's esophagus. Gastroenterology 2002;122:1101-1112.

40 Fein M, Peters JH, Baril N, et al. Loss of function of Trp53, but not Apc, leads to the development of esophageal adenocarcinoma in mice with jejunoesophageal reflux. J Surg Res 1999;83:48-55. 